\title{
Human Parasitic Diseases in Bulgaria in Between 2013-2014
}

\author{
Iskra Rainova, Rumen Harizanov, Iskren Kaftandjiev, Nina Tsvetkova, Ognyan Mikov, Eleonora Kaneva
}

Department of Parasitology and Tropical Medicine, National Centre of Infectious and Parasitic Diseases, Sofia, Bulgaria

Background: In Bulgaria, more than 20 autochthonous human parasitic infections have been described and some of them are widespread. Over 50 imported protozoan and helminthic infections represent diagnostic and therapeutic challenges and pose epidemiological risks due to the possibility of local transmission.

Aims: To establish the distribution of autochthonous and imported parasitic diseases among the population of the country over a 2-year period (2013-2014) and to evaluate their significance in the public health system.

Study Design: Cross sectional study.

Methods: We used the annual reports by regional health inspectorates and data from the National Reference Laboratory at the National Centre of Infectious and Parasitic Diseases on all individuals infected with parasitic diseases in the country. Prevalence was calculated for parasitic diseases with few or absent clinical manifestations (oligosymptomatic or asymptomatic infections). Incidence per 100.000 was calculated for diseases with an overt clinical picture or those that required hospitalisation and specialised medical interventions (e.g. surgery).
Results: During the research period, parasitological studies were conducted on 1441.244 persons, and parasitic infections were diagnosed in 22.039 individuals. Distribution of various parasitic pathogens among the population displayed statistically significant differences in prevalence for some intestinal parasites (enterobiasis $0.81 \%$, giardiasis $0.34 \%$ and blastocystosis $0.22 \%$ ). For certain zoonotic diseases such as cystic echinococcosis (average incidence of 3.99 per 100.000) and trichinellosis (average incidence of 0.8 per 100.000), the incidence exceeds several times the annual incidence recorded in the European Union.

Conclusion: Parasitic diseases still pose a substantial problem with social and medical impacts on the residents of our country. Improved efficiency regarding autochthonous and imported parasitic diseases is essential in providing the public health system the tools it needs to combat these diseases. Attention should be focused on the various imported vector-borne parasitic diseases (e.g. malaria and cutaneous leishmaniasis) for which the country is potentially endemic.

Keywords: Parasitic disease, prevalence, incidence, transmission, Bulgaria 
According to the World Health Organization (1), over 3 billion people around the world suffer from one or more parasitic diseases, and in most tropical or subtropical countries, they are widespread and a leading cause for morbidity and mortality in the population. In Bulgaria, more than 20 human parasitic infections with autochthonous distribution have been described and some of them are widespread: enterobiasis, hydatid disease, and trichinellosis (2). Growing relationships with tropical and subtropical countries increase the risk of inadvertently importing tropical parasitic diseases. Over 50 imported protozoan and helminthic infections represent diagnostic and therapeutic challenges and pose epidemiological risk due to the possibility of local transmission. More than 2 million Bulgarians travel annually across all regions of the world (3). The geopolitical changes in recent years have led to strong migratory pressure on European countries (including Bulgaria) due to migrants coming from the Middle East and North and sub-Saharan Africa. This mass movement of people increases the risk of the introduction and spread of parasitic pathology that currently has no local distribution.

The aim of this study was to conduct a retrospective epidemiological analysis on the prevalence of autochthonous and imported human parasitic diseases in Bulgaria for a period of 2 years (2013-2014) and to evaluate their significance on the public health system in the country.

\section{MATERIALS AND METHODS}

The use of these data was consistent with the regulations of the National Centre of Infectious and Parasitic Diseases (NCIPD), Sofia, Bulgaria (Approval number: 1000-107/20.02.17)

\section{Surveillance system}

Under current regulations, 11 parasitic diseases are subject to mandatory reporting and registration: ascariasis, cryptosporidiosis, congenital toxoplasmosis, echinococcosis, giardiasis, hymenolepiasis, malaria, taeniasis, trichinellosis, trichuriasis and visceral leishmaniasis (4). Surveillance and control of all parasitic diseases is carried out in accordance with the Ministry of Health $(\mathrm{MoH})$ regulation regarding 'the diagnosis, prevention and control of local parasitic diseases' (5). Data on diagnosed cases are provided to the respective administrative district by the Departments of Medical Parasitology and Epidemiology at the Regional Health Inspectorates (RHI). In the Department of Parasitology and Tropical Medicine at the NCIPD, the information is summarised, analysed and presented to the $\mathrm{MoH}$.

\section{Patients and samples}

According to the country's legislation, the following groups of people undergo an annual mandatory check-up for intestinal parasites: children attending nurseries and kindergartens and the personnel working in them, the staff in public food services, persons working in the field of production and trade of food products, and migrants and refugees who arrived in Bulgaria.

Our survey includes all Bulgarian and foreign nationals residing in the country who were examined for parasitic diseases from January 2013 to December 2014.

\section{Parasitological diagnosis}

In Bulgaria, parasitological diagnosis is carried out in 89 laboratories: 28 laboratories at RHIs and 61 independent medical diagnostic laboratories or laboratories in hospitals based in the 28 districts of the country. Routine and confirmatory diagnostic activities are carried out at the National Reference Laboratory (NRL) at NCIPD.

Stool specimens were examined for intestinal helminths and protozoa by various methods: direct wet smear, Lugol's iodine, formalin-ether and other concentration techniques and culture of larval-stage nematodes. For detection of Enterobius vermicularis eggs, the transparent tape test was used. For intestinal protozoa, staining techniques, culture methods and rapid immunochromatographic tests were used. For blood and tissue parasites, the following techniques were used: staining for examination of blood and bone marrow smears, culture methods, detection of specific antibodies by ELISA, Western blot, rapid immunochromatographic tests and molecular analysis by polymerase chain reaction (PCR). Immunological diagnosis and PCR were performed with certified, CE-marked commercial kits (Table 1).

\section{Data collection}

We used the annual reports by RHIs on all individuals infected with parasitic diseases in the country and data from the NRL at NCIPD. Data from the National Statistical Institute on the country's population size was also collected and these data were used to calculate parameters such as prevalence and incidence. Prevalence was calculated for parasitic diseases with few or absent clinical manifestations (oligosymptomatic or asymptomatic infections), and incidence per 100.000 was calculated for diseases with overt clinical picture or requiring hospitalisation and highly specialised medical care (e.g. surgery).

\section{RESULTS}

During the research period, parasitological examinations were conducted on 1441.244 persons (713.446 or $10.2 \%$ of the population in 2013 and 727.798 or $9.9 \%$ of the population in 2014). A total of 22.039 (1.53\%) patients were diagnosed with parasitic diseases $(n=10.310,1.44 \%$ for $2013, n=11.729,1.61 \%$ 
for 2014) (Table 2). Diagnosis was confirmed by microscopic methods in $68 \%$ of the cases and in $32 \%$ by immunological or molecular methods.

\section{Diseases with local transmission}

Cystic echinococcosis: A total of 639 cases of hydatid disease were recorded. The relative share of primary cases was $90.85 \%$ $(n=278$ for 2013) and 92.69\% $(n=302$ for 2014). Average incidence of hydatid disease in 2013 was 3.82 per 100.000, and 4.17 per 100.000 in 2014. The most affected groups were patients 20-29 years old (14\%), 30-39 years old and 60-69 years old (13\% both groups). Among children and adolescents (0-18 years), $113(17.7 \%)$ cases of the disease $(n=55$ for 2013 and $\mathrm{n}=58$ for 2014) were recorded. In $2014,12(20.69 \%)$ of the

TABLE 1. Methods used for laboratory diagnosis

(according to the 'Medical Parasitology' medical standard issued by the Ministry of Health of the Republic of Bulgaria 23.12.2014)

\begin{tabular}{|c|c|}
\hline Diseases & Parasitological methods used for laboratory diagnosis \\
\hline Cystic echinococcosis & $\begin{array}{l}\text { Commercial (IgG ELISA); microscopic examination of hydatid fluid and Nakanishi method; commercial WB*; PCR } \\
\text { methods for species identification of E. granulosus* }\end{array}$ \\
\hline Trichinellosis & Commercial IgG ELISA; commercial WB*; PCR methods for species identification of Trichinella spp. \\
\hline Taeniasis & $\begin{array}{l}\text { Macroscopic examination of the gravid proglottids; microscopic examination of the gravid proglottids; microscopic } \\
\text { examination of transparent tape test }\end{array}$ \\
\hline Cysticercosis & $\begin{array}{l}\text { Microscopic examination for presence of suckers and hooks on the scolex after surgical removal; commercial ELISA; } \\
\text { commercial WB* }\end{array}$ \\
\hline Toxocariasis & Commercial ELISA; commercial WB* \\
\hline Toxoplasmosis & Commercial ELISA IgG, IgM, IgA*; IgG avidity*; PCR* \\
\hline Visceral leishmaniasis & $\begin{array}{l}\text { Microscopic examination of bone marrow aspirates; commercial ELISA; commercial WB*; commercial rapid } \\
\text { immunochromatographic tests for detection of anti-rK-39 antibodies; culture (NNN)*; PCR* }\end{array}$ \\
\hline Soil-transmitted helminth infections & Microscopic examination of eggs after formalin-ether and other concentration techniques; macroscopic examination \\
\hline Enterobiasis & Transparent tape test \\
\hline Giardiasis & Direct wet smear; Lugol's iodine; commercial rapid immunochromatographic tests; PCR* \\
\hline Hymenolepiasis & Microscopic examination of eggs after formalin-ether and other concentration techniques \\
\hline Cryptosporidiosis & Commercial rapid immunochromatographic tests; modified Ziehl-Neelsen acid-fast stain* \\
\hline Blastocystosis & Direct wet smear; Lugol's iodine; culture; PCR* \\
\hline Malaria & Thick blood films; thin blood films; commercial rapid immunochromatographic tests; PCR* \\
\hline Cutaneous leishmaniasis & $\begin{array}{l}\text { Microscopic examination of Giemsa-stained touch preparations from aspirates and/or biopsy specimens; culture (NNN)*; } \\
\text { PCR* }\end{array}$ \\
\hline Filariasis & Knott concentration procedure*; thick blood films; thin blood films; commercial rapid immunochromatographic tests* \\
\hline Schistosomiasis & Microscopic examination of stool or urine probes; commercial rapid immunochromatographic tests*; commercial ELISA \\
\hline
\end{tabular}

TABLE 2. Data on registered autochthonous human parasitic diseases in Bulgaria 2013-2014

\begin{tabular}{|c|c|c|c|c|}
\hline \multirow[b]{2}{*}{ Indigenous parasitic diseases } & \multicolumn{2}{|c|}{ Year } & \multirow[b]{2}{*}{ Total } & \multirow[b]{2}{*}{ Mean } \\
\hline & 2013 & 2014 & & \\
\hline Cystic echinococcosis & 306 & 333 & 639 & 319.5 \\
\hline Trichinellosis [Rainova et al. (19) 2016] & 60 & 67 & 127 & 63.50 \\
\hline Taeniosis & 36 & 23 & 59 & 29.50 \\
\hline Toxoplasmosis $\operatorname{IgM}^{+} \operatorname{IgG}^{+}$ & 74 & 103 & 177 & 88.50 \\
\hline Toxocariasis & 59 & 31 & 90 & 45.00 \\
\hline Ascariasis & 473 & 666 & 1139 & 569.5 \\
\hline Trichiuriasis & 93 & 100 & 193 & 96.50 \\
\hline Enterobiasis & 3752 & 4842 & 8594 & 4297 \\
\hline Giardiasis & 1873 & 1731 & 3604 & 1802 \\
\hline Hymenolepiasis & 463 & 201 & 664 & 332.0 \\
\hline Blastocystosis & 1355 & 857 & 2212 & 1106 \\
\hline Cryptosporidiosis & 1 & 2 & 3 & 1.500 \\
\hline
\end{tabular}


infected children were in the 0-4 year age group. Two deaths due to cystic hydatid disease (4-year-old boy and 48-year-old woman) were recorded. The highest incidence was registered in the region of Sliven with average incidence of 14 per 100.000 for 2013 and 2014, the region of Kardzhali with 12 per 100.000 and the region of Shumen with 9.8 per 100.000 (Figure 1).

Trichinellosis: Seven outbreaks (two in 2013 and five in 2014) were recorded in the country. A total of 261 persons consumed meat products contaminated with Trichinella spiralis larvae. Infection confirmed by laboratory tests (ELISA and Western blot) was established in 127 (48.66\%) of the cases. Clinical symptoms developed in $123(96.85 \%)$ patients and $4(3.15 \%)$ remained asymptomatic. Out of the 127 people infected in both years, $60(47.24 \%)$ were men, $46(36.22 \%)$ were women and $21(16.54 \%)$ were children and adolescents (6). In 4 of the outbreaks, the source of infection was meat from wild boar, 2 of the outbreaks could be attributed to the domestic pig, and in 1 outbreak, the source was mixed meat from wild boar and domestic pig. The average incidence of trichinellosis in the country was 0.8 per 100.000 . Outbreaks were recorded in the regions of Sofia, Sofia-capital, Plovdiv, Dobrich and Burgas (Figure 1). The outbreaks associated with consumption of meat from wild boar were caused by Trichinella britovi, while those with meat from domestic pig by $T$. spiralis.

Taeniasis: During the study period, 59 cases of taeniasis caused by Taenia saginata were recorded. The incidence was 0.49 per 100.000 for 2013 and 0.32 per 100.000 for 2014 . Autochthonous cases of intestinal Taenia solium infections were not registered, but 36 serologic tests for cysticercosis were conducted at NCIPD by clinical indications and positive results in ELISA were found in 2 patients with clinical manifestations of neurocysticercosis.

Toxocariasis: Four hundred sixty-six persons were examined for toxocariasis by serological tests using ELISA and Western blot, primarily due to peripheral blood eosinophilia and/or allergy symptoms. Positive test results were found in 90 patients (19.3\%).

Toxoplasmosis: Tests for toxoplasmosis were conducted on pregnant women or women enrolled in programmes for in vitro fertilisation, patients with lymphadenitis, patients with vague febrile illness, patients with inflammation of the eye and immunocompromised patients with low CD4 cell counts. A total of 19.823 individuals were studied ( $\mathrm{n}=10.368$ in 2013 and $\mathrm{n}=9.455$ in 2014). The presence of specific immunoglobulin $\mathrm{G}(\mathrm{IgG})$ antibodies was found in 3.962 (19.97\%) of the tested persons, anti-Toxoplasma IgG and immunoglobulin M (IgM) antibodies in $177(0.89 \%)$ patients, and $33(0.17 \%)$ persons were positive for $\operatorname{IgG}$, IgM and immunoglobulin A. IgG avidity was examined in 32 patients, and of these, 13 were pregnant women in different stages of gestation. Low avidity was confirmed in 3 of the pregnant women

Visceral leishmaniasis: Twenty-seven cases of visceral leishmaniasis caused by Leishmania infantum were recorded in the country. Twenty-five of them were autochthonous and 2 were imported from Portugal and Greece. Twelve of the cases were in children, and 15 were in adults. Established incidence for the period was 0.19 per 100.000 .

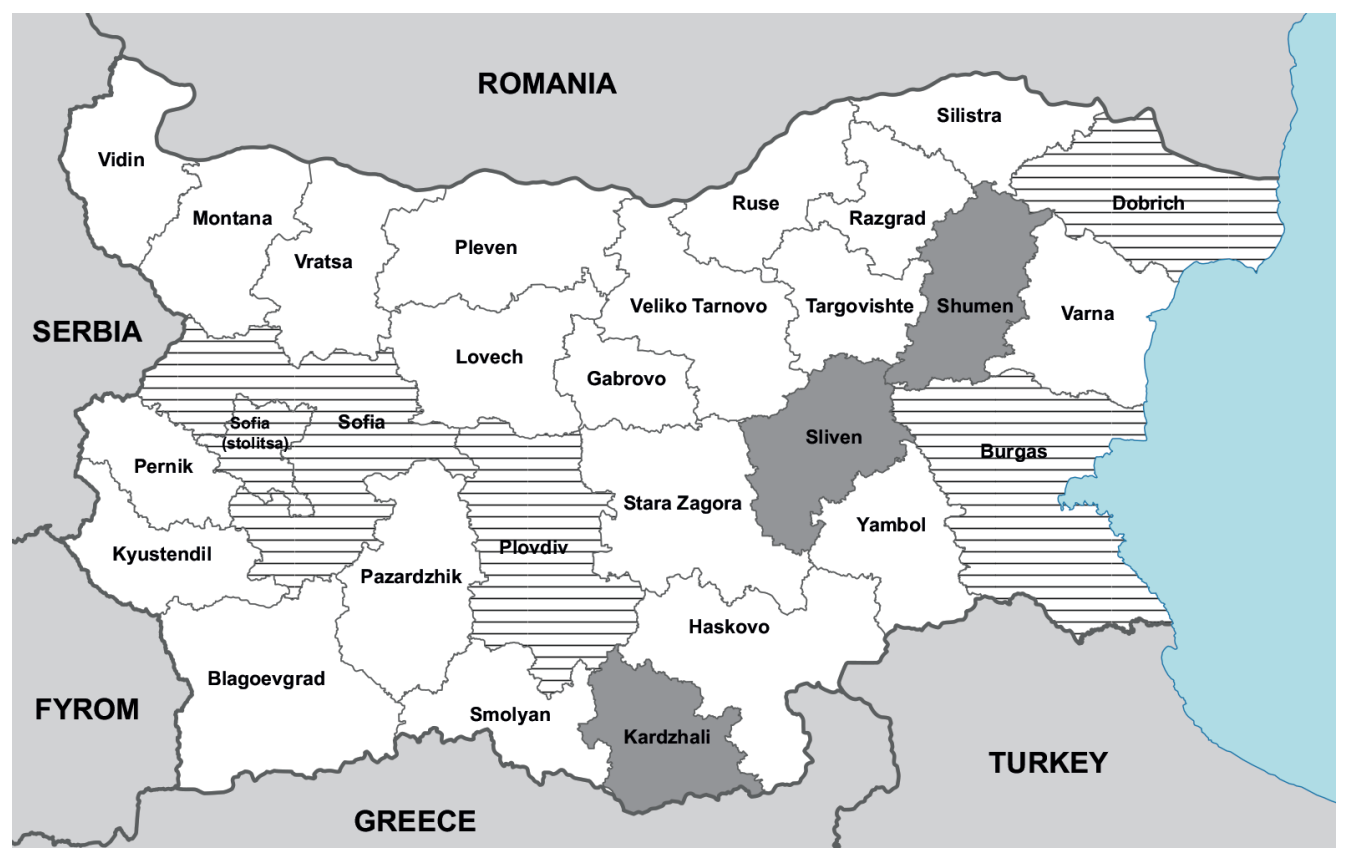

FIG. 1. Administrative map of Bulgaria. The districts with higher incidence of cystic echinococcosis are marked with a solid fill. The areas with outbreaks of trichinellosis recorded during the studied period are marked with hatched lines. 
Soil-transmitted helminth infections: Ascariasis and trichuriasis have local transmission in Bulgaria. A total of 1 170.966 persons $(n=589.417$ in 2013 and $n=581.549$ in 2014) were examined. Of them, 1139 persons were positive for ascariasis, with an average incidence of 7.84 per 100.000 for the period. Also, 193 cases of trichuriasis were recorded with an average incidence of 1.37 per 100.000 for the period.

\section{Communicable parasitic diseases}

Enterobiasis: During the study period, 1056.805 persons (500.251 in 2013 and 556.554 in 2014) were examined. The average national prevalence for the period was $0.81 \%(0.75 \%$ in 2013 and $0.87 \%$ in 2014). Annually, more than $90 \%$ of the children in childcare facilities were examined for enterobiasis. During the studied period, 353.106 children aged 1-7 years were residing in different types of childcare. The established average prevalence of $1.1 \%$ in this group was higher than the national average.

Giardiasis: A total of 1062.600 persons (positive $n=3604$ ) were tested for giardiasis, and the average prevalence was $0.34 \%$ ( $0.36 \%$ in 2013 and $0.31 \%$ in 2014).

Hymenolepiasis: A total of 911.922 people were examined for hymenolepiasis and 664 of them were infected. The average prevalence for the period was $0.07 \%$.

\section{Other intestinal protozoan infections}

For cryptosporidiosis, 841 people were tested. Three patients $(0.36 \%)$ were positive and 1 of them was also HIV positive. For blastocystosis 1005.828 persons (501.800 in 2013, and 504.028 in 2014) were examined, with an average prevalence in the country of $0.22 \%(n=2212)$.

\section{Imported parasitic diseases}

During the 2-year study period, 8.970 persons were examined, including 611 Bulgarian citizens (Table 3). According to the

TABLE 3. Data on recorded imported parasitic diseases in Bulgaria 2013-2014

\begin{tabular}{lcccc}
\hline \multirow{2}{*}{ Imported parasitic diseases } & \multicolumn{3}{c}{ Year } & \\
\cline { 2 - 3 } Falciparum malaria & 2013 & 2014 & Total & Mean \\
Vivax malaria & 5 & 9 & 14 & 7 \\
Cutaneous leishmaniasis & 3 & 1 & 4 & 2 \\
Loiasis & 0 & 2 & 2 & 1 \\
Urogenital schistosomiasis & 1 & 0 & 1 & 0.5 \\
Ascariasis & 1 & 0 & 1 & 0.5 \\
Trichuriasis & 0 & 6 & 6 & 3 \\
Giardiasis & 0 & 1 & 1 & 0.5 \\
Enterobiasis & 23 & 28 & 51 & 25.5 \\
Hymenolepiasis & 6 & 5 & 11 & 5.5 \\
Blastocystosis & 4 & 4 & 8 & 4 \\
\hline
\end{tabular}

current legislation, all persons accommodated in refugee camps in Bulgaria must be tested for intestinal parasites and those coming from endemic countries should also be tested for malaria. This is carried out by RHIs working in each of the 28 districts of the country.

Malaria: Bulgaria is certified as being malaria-free since 1965. A total of 3.838 persons were tested for malaria, including 553 Bulgarian citizens. Eighteen cases of imported malaria were diagnosed: 8 in 2013 and 10 in 2014. Most of the diseased were Bulgarian citizens $(n=14)$. Plasmodium falciparum was identified as the causative agent in 14 cases and Plasmodium vivax in 4.

Intestinal parasitic diseases: Prophylactic examinations for intestinal parasites were conducted in 6.147 foreign nationals who sought asylum in the country. The examinations found the presence of the following parasites: Giardia intestinalis, Blastocystis hominis, Enterobius vermicularis, Hymenolepis nana, Ascaris lumbricoides, and Trichocephalus trichiurus (Table 3).

Other imported parasitic diseases: Two cases of cutaneous leishmaniasis, 1 case of loiasis and 1 case of urogenital schistosomiasis were diagnosed during the period, all of which do not have autochthonous transmission in Bulgaria.

\section{DISCUSSION}

\section{Parasitic infections with local transmission}

During the research period, around $10 \%$ of the country's population was examined for parasitic infections. Two of these, including cystic echinococcosis (CE) and trichinellosis, have important medical and public health implications. A disturbing fact is the high proportion of echinococcosis among children and adolescents $(17.7 \%, \mathrm{n}=113)$, which indicates a high transmission rate in the country (7-9). The incidence of cystic hydatid disease in the European Union for the reviewed period was 0.18 per $100.000(10,11)$, but in Bulgaria the incidence is 30-40 times higher. Possible reasons for the high incidence of human $\mathrm{CE}$ in Bulgaria is the large number of free-ranging stray dogs and the breeding of yard and shepherd dogs in some rural areas that are not regularly dewormed which, in turn, leads to a high infection rate among domestic animals (7). In a study of the prevalence of echinococcosis among livestock at slaughter meat production houses, animals that were most affected were the sheep $(6.4 \%)$, followed by cattle $(4.3 \%)$, goats $(0.9 \%)$ and pigs (less than $0.1 \%)(7,12)$.

After the political and economic changes, human trichinellosis became a re-emerging zoonosis in Bulgaria since 1991. Between 1990 and 2006, 145 trichinellosis outbreaks and 238 sporadic cases were recorded (13). In 2013-2014, 536 cases of 
human trichinellosis $(10,11)$ were recorded in the EU, of which $130(24.3 \%)$ were registered in Bulgaria. Accordingly, the incidence was 0.05 (2013) and 0.07 (2014) per 100.000 for the $\mathrm{EU}$, and 0.82 and 0.83 per 100.000 for Bulgaria (6). Regarding the Trichinella spp. causing outbreaks in the country, some previous (14) and recent studies (6) confirmed the invariable presence of 2 species, T. britovi and T. spiralis.

In Bulgaria, toxocariasis is not a notifiable disease, but according to previous studies of patients with allergy and/or eosinophilia, its seroprevalence ranges between $12.25 \%$ and $14.01 \%$, (15-18). Our present survey of patients with allergy and/or eosinophilia established a seroprevalence of $19.3 \%$, while earlier research (2005-2008) conducted among healthy blood donors $(n=350)$ found a seroprevalence of $3.4 \%$ (19). Based on the aforementioned data, it can be approximated that the average prevalence of toxocariasis among persons with allergy and/or eosinophilia is $15.09 \%$, which is approximately 5 times more than healthy people.

Although our study was not a screening of the Bulgarian population for toxoplasmosis, the seroprevalence values we found were similar to the those established by previous studies in Bulgaria (24.1\% for 2011 and $20 \%$ for 2012) (18), and is slightly lower than values found in a 2004 Greek study that established seroprevalence among the general population as $24.1 \%$ (20). According to some authors, approximately $25 \%$ $30 \%$ of the world human population is infected by Toxoplasma spp. (21). Actually, the prevalence varies wildly between countries (from $10 \%$ to $80 \%$ ) and often within a given country or between different communities in the same region (22). Over the 2013-2014 time period, primary toxoplasmosis was diagnosed in 13 pregnant women at NCIPD. Three of them had low IgG avidity. In pregnant women, it is not always easy to date the infection versus the time of conception, but a high avidity result in early pregnancy can generally help rule out infection in pregnancy in most cases of positive specific IgM.

Soil-transmitted helminth infections (ascariasis and trichuriasis) can be defined as 'habitual' for the country, because cases among the population are recorded every year at approximately the same level. Analysis of the data has shown that in recent years the total number of people infected with $A$. lumbricoides varies between 500 and 700 per year and for people infected with $T$. trichiurus, about 100 per year. Regions with the greatest number of ascariasis cases are in the southern part of the country where the climate is more suitable for parasite transmission. Trichuriasis is registered more often among people living in social institutions (for children deprived of parental care and adults in mental care facilities) (23). Ancylostomiasis is not endemic to the country and is without local transmission.

The communicable parasitic diseases are of particular health importance for Bulgaria because they affect mostly children in organised groups. Of these diseases, enterobiasis is still the most common and its prevalence levels remain high (24). Although the prevalence of giardiasis shows a declining trend, it retains its medical importance, while cases of hymenolepiasis are rarely recorded.

Cases of cryptosporidiosis in people are seldom diagnosed, although a pilot study employing immunofluorescence and PCR techniques proved the presence of Cryptosporidium spp. oocysts in various sources of drinking water (25). The low incidence of cryptosporidiosis among humans in the country is probably due to the relatively mild clinical symptoms in immunocompetent persons and to the relatively low number of HIV-positive persons ( $\mathrm{n}=2046$ ) (26).

Since 1989, autochthonous cases of visceral leishmaniasis caused by Leishmania infantum are annually registered in Bulgaria. By 2012, 122 cases of the disease were recorded, of which 118 were indigenous and 4 imported cases were from leishmaniasis-endemic European countries (Italy and Spain) (27). During this study, most of the autochthonous cases of visceral leishmaniasis were recorded in 2 municipalities (Petrich and Parvomay) located in southern Bulgaria, and 2 cases were imported from Greece and Portugal.

\section{Imported parasitic diseases}

Over the course of our study, we observed imported parasitic diseases such as malaria, cutaneous leishmaniasis, urogenital schistosomiasis and loiasis, none of which had local transmission in the country. The rest of the imported cases were intestinal parasitic diseases such as soil-transmitted helminth infections (ascariasis and trichuriasis), communicable parasitic diseases (enterobiasis, hymenolepiasis, giardiasis, and blastocystosis) and visceral leishmaniasis, all of which have local distribution. The gathering of large groups of migrants with such parasitic infections in endemic areas may lead to formation of new foci of transmission or to an increase in the intensity of the pre-existing ones. It seems that the import of parasitic diseases from endemic regions will continue in the coming years. This influx is associated with expanding trade, political and economic relations, tourism and, unfortunately, mass migration of people. The country has favourable climate and fauna conditions for local distribution of a number of parasitic diseases. In 1995 a local outbreak of imported malaria that reported illegal immigrants from Afghanistan as a source was recorded (28). Similar cases have been reported in Greece between 2009 and 2013 with registered cases of tertian malaria with local transmission imported by migrants (29).

The local transmission of parasitic diseases directly depends on the effectiveness of the system for surveillance and control. 
Regardless of the improvement of living standards and tools for health education, parasitic diseases are a significant part of the overall pathology registered in Bulgaria. It is essential to constantly improve the efficiency of the public health system in order to deal with autochthonous parasitic diseases. It is also imperative to maintain a state of increased preparedness for action against tropical parasitic diseases that may be imported more often into the country in the coming years.

Financial Disclosure: No financial disclosure was declared by the authors.

Conflict of Interest: No conflict of interest was declared by the authors.

\section{REFERENCES}

1. WHO/Department of Control of Neglected Tropical Diseases. Sustaining the drive to overcome the global impact of neglected tropical diseases: second WHO report on neglected diseases. Geneva; 2013.

2. Boeva-Bangyosova V, Vutova K. Parasitology (local and tropical parasitic diseases). Sofia: Arso; 2008.

3. National statistical institute. Trips of Bulgarian residents abroad by months and by country of destination. (cited 2016 April 7) Available from: http:// www.nsi.bg/en/content/7054/trips-bulgarian-residents-abroad-monthsand-country-destination.

4. Ministry of Health. Ordinance no. 21/08.07.2011 for the registration, notification and reporting of communicable diseases. State Gazette 2011;52:29-61.

5. Ministry of Health. Ordinance no. 5/06.04.2006 on the diagnosis, prevention and control of local parasitic diseases. State Gazette 2006;40:83-90.

6. Rainova I, Kaftandjiev I, Harizanov R, Tsvetkova N, Jordanova D, Marinova I, et al. Outbreaks of human trichinellosis, still a challenge for the public health authorities in Bulgaria. J Publ Health 2016;24:2917.

7. Jordanova DP, Harizanov RN, Kaftandjiev IT, Rainova IG, Kantardjiev TV. Cystic echinococcosis in Bulgaria 1996-2013, with emphasis on childhood infections. Eur J Clin Microbiol Infect Dis 2015;34:1423-8.

8. Todorov T, Boeva V. Echinococcosis in children and adolescents in Bulgaria: a comparative study. Ann Trop Med Parasitol 2000;94:135-44.

9. Vlad DC, Neghina AM, Dumitrascu V, Marincu I, Neghina R, Calma CL. Cystic echinococcosis in children and adults: a seven-year comparative study in western Romania. Foodborne Pathog Dis 2013;10:189-95.

10. European Food Safety Authority and European Centre for Disease Prevention and Control. The European Union summary report on trends and sources of zoonoses, zoonotic agents and food-borne outbreaks in 2013. EFSA Journal 2015;13:3991.

11. European Food Safety Authority and European Centre for Disease Prevention and Control. The European Union summary report on trends and sources of zoonoses, zoonotic agents and food-borne outbreaks in 2014. EFSA Journal 2015;13:4329.
12. European Food Safety Authority and European Centre for Disease Prevention and Control. The European Union summary report on trends and sources of zoonoses, zoonotic agents and food-borne outbreaks in 2010. EFSA Journal 2012;10:2597.

13. Kurdova-Mintcheva R, Jordanova D, Ivanova M. Human trichinellosis in Bulgaria--epidemiological situation and trends. Vet Parasitol 2009;159:3169.

14. Kurdova R, Müller N, Tsvetkova N, Michov L, Georgieva D, Ivanova M, et al. Characterisation of Trichinella isolates from Bulgaria by molecular typing and cross-breeding. Vet Parasitol 2004;123:179-188.

15. Rainova I. Toxocariasis. In: Kurdova R, ed. Parasitic zoonoses in humans. Sofia: NCIPD; 2008:88-110.

16. Kurdova R, Petrov P, Jordanova D, Rainova I, Harizanov R, Marinova I, et al. Parasitic diseases in Bulgaria 2007 - 2008. Status and prognosis. NCIPD Informational Journal 2010;5:5-30.

17. Kurdova R, Rainova I, Jordanova D, Harizanov R, Marinova I, Bikov I, et al. Characterization of parasitic incidence in Bulgaria in 2010. NCIPD Informational Journal 2011;2:5-29.

18. Rainova I, Marinova I, Harizanov R, Jordanova D, Kaftandjiev I, Bikov I, et al. Parasitic diseases in Bulgaria in 2012. Probl Inf Parasit Dis 2014;42:29-38.

19. Rainova I. Study of Toxocara antibody bearing among healthy people in Bulgaria. In MEDIMOND International Proceedings $X$ European Multicolloquim of Parasitology (EMOP X), eds. Jean Dupouy-Camet and Eduardo Dei-Cas. Free papers. Paris (France), 2008:8;115-118. Available at: http://www.monduzzi.com/proceedings/moreinfo/20080824.htm.

20. Diza E, Frantzidou F, Souliou E, Arvanitidou M, Gioula G, Antoniadis A. Seroprevalence of Toxoplasma gondii in northern Greece during the last 20 years. Clin Microbiol Infect 2005;11:719-23.

21. Montoya JG, Liesenfeld O. Toxoplasmosis. Lancet 2004;363:1965-76.

22. Pappas G, Roussos N, Falagas ME. Toxoplasmosis snapshots: global status of Toxoplasma gondii seroprevalence and implications for pregnancy and congenital toxoplasmosis. Int J Parasitol 2009;39:1385-94

23. Harizanov R, Rainova I, Kaftandjiev I, Jordanova D, Marinova I. Soiltransmitted helminth infections in Bulgaria: a retrospective study of some epidemiological features. Probl Inf Parasit Dis 2013;41:32-5.

24. Rainova I, Harizanov R, Jordanova D, Tsvetkova N, Marinova I. State and distribution of enterobiasis in Bulgaria for the period from 2008 to 2012. In: Proceedings of the Twelfth National Congress of Clinical Microbiology and Infections. Sofia: Bulgarian Association of Microbiologists, 2014:42 (abstract).

25. Karanis P, Sotiriadou I, Kartashev V, Kourenti C, Tsvetkova N, Stojanova $\mathrm{K}$. Occurrence of Giardia and Cryptosporidium in water supplies of Russia and Bulgaria. Environ Res 2006;102:260-71.

26. National Program for Prevention and Control of HIV/AIDS "at the Ministry of Health. HIV/AIDS data. Available from: http://www.aidsprogram.bg/ static_info1.php?main=data

27. Harizanov R, Rainova I, Tzvetkova N, Kaftandjiev I, Bikov I, Mikov O. Geographical distribution and epidemiological characteristics of visceral leishmaniasis in Bulgaria, 1988 to 2012. Euro Surveill 2013;18:205-31.

28. Kurdova R, Vuchev D, Petrov P. Malaria situation in Bulgaria and surveillance measures (1991-2000). Global Nest: the Int J 2001;3:153-62.

29. Hellenic Center for Diseases Control and Prevention. Epidemiological surveillance report Malaria in Greece. (cited 2016 February 10) Available from: http//www.keelpno.gr/.../Malaria\%20annual\%20report. 